INL/EXT -17-43386

September 2017

\title{
Development of a fully validated quantitative model of hydride morphology in zirconium alloy nuclear fuel cladding
}

Pierre-Clement Simon Michael Tonks Arthur Motta Long-Qing Chen

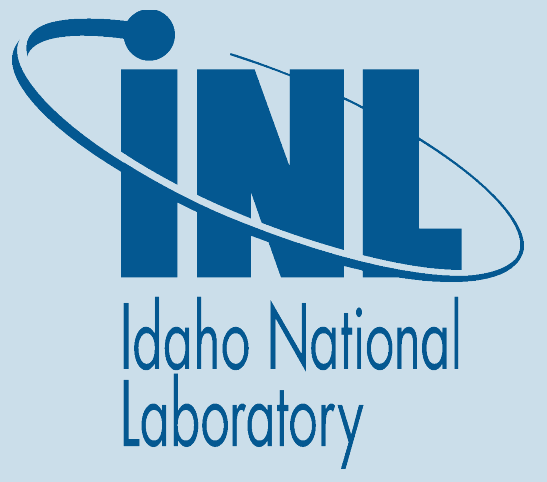




\section{NOTICE}

This information was prepared as an account of work sponsored by an agency of the U.S.

Government. Neither the U.S. Government nor any agency thereof, nor any of their employees, makes any warranty, express or implied, or assumes any legal liability or responsibility for any third party's use, or the results of such use, of any information, apparatus, product, or process disclosed herein, or represents that its use by such third party would not infringe privately owned rights. The views expressed herein are not necessarily those of the U.S. Nuclear Regulatory Commission. 
Development of a fully validated quantitative model of hydride morphology in zirconium alloy nuclear fuel cladding

\author{
Pierre-Clement Simon \\ Michael Tonks \\ Arthur Motta \\ Long-Qing Chen*
}

September 2017

The Pennsylvania State University Department of Nuclear Engineering University Park, PA

"The Pennsylvania State University Department of Materials Science and Engineering University Park, PA

Prepared for the U.S. Department of Energy Office of Nuclear Energy Under U.S. Department of Energy-Idaho Operations Office Contract DE-AC07-99ID13727 


\section{Abstract}

The precipitation of hydrogen in zirconium cladding can compromise the safety of nuclear facilities. A phase field model is being developed to study the precipitation of hydride, their orientation and their morphology. A multi-phase field model and a grand potential model were developed and are coupled with elastic models using the properties of the alpha zirconium and delta hydride phase to study the precipitation of hydrides, their orientation and their morphology. Both models were verified using analytical solutions, and then applied to the $\alpha-\mathrm{Zr} / \delta-\mathrm{ZrH}_{1.66}$ system. Future work will take plasticity into account to study sympathetic nucleation.

\section{Contents}

\begin{tabular}{lll}
\hline 1 & Introduction & 2
\end{tabular}

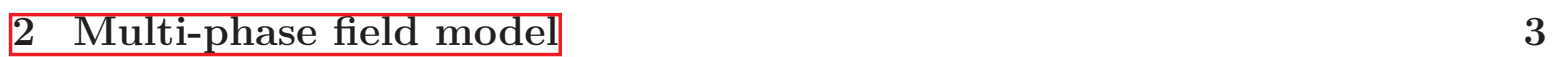

2.1 Generalities . . . . . . . . . . . . . . . . . . . . . 3

2.2 Total free energy $\ldots \ldots \ldots \ldots \ldots \ldots$

2.3 Phase field equations . . . . . . . . . . . . . . . . . . . . . . . . . . . 4

2.3 .1 Cahn-Hilliard equation, for conserved variables . . . . . . . . 5

2.3.2 Allen-Cahn equation, for non-conserved variables . . . . . . . . 5

3 Grand potential model 5

3.1 Generalities . . . . . . . . . . . . . . . . . . . . . . 5

3.2 Expression of the grand potential . . . . . . . . . . . . . . . . . . . . . 6

3.3 Grand potential models equations . . . . . . . . . . . . . . . . 6

\begin{tabular}{|lll}
4 & Coupling with elasticity & 7
\end{tabular}

4.1 Generalities . . . . . . . . . . . . . . . . . . . . . . . . . 7

4.2 VTS and KHS models . . . . . . . . . . . . . . . . . . . . . . . . . . . 7

4.3 Simulations results, and comparison with analytical solution . . . . . . 7 
5 Application to the Zirconium hydride model

Conclusion and future work

\section{Introduction}

Zirconium alloys are widely used in the nuclear industry. Due to their properties, they are the current choice for the fuel cladding in the reactor core for $\mathrm{UO}_{2}$ fuel in Light Water Reactors. A zirconium cladding indeed presents a low neutron absorption cross section, a good thermal conductivity, and an adequate resistance to corrosion. It also resists well to void swelling, and its mechanical properties are compatible with the ones from the fuel. Moreover, zirconium is available at a reasonable cost. However, the precipitation of hydrogen in the zirconium can cause problems during operation or dry storage. These hydrides cause the embrittlement of the cladding, which facilitates crack propagation, and thus leads to the release of fission products. Understanding hydride precipitation would increase the safety of nuclear facilities by preventing cladding failure.

An abundance of experimental research has been conducted to study zirconium hydrides ([1], 2, [3]), but a quantitative numerical model is still needed to study the effect of the different physics involved. The multi-phase field model is a method of choice to study microstructure evolution ([4, 5] ), and this model is presented in section

2. However, the grand potential model has the great advantage of decoupling the bulk free energy contribution to the interfacial free energy of the system, thus potentially reducing the computational time. Its formulation is detailed in section 3. The coupling of these models to elastic models is described in section 4 . Finally, the application of these phase field model to the zirconium hydride system is described in section 5 . 


\section{Multi-phase field model}

\subsection{Generalities}

A multi-phase field model describes the change in composition as well as the evolution of the structural aspect of a microstructure using field variables ([6, 7, 5] ). These field variables are divided into two types, conserved and non-conserved. Conserved variables, like concentration, must respect the conservation equation, while non-conserved variables, like order parameters, do not. All variables are continuous across the entire domain. As a consequence, the interfaces of the microstructure are diffuse. This aspect of phase field models makes it sometimes challenging to analyze since interfaces are usually descrete in real materials, but it allows the study the evolution of microstructures by simply solving partial differential equations.

In the case of the $\mathrm{Zr} / \mathrm{H}$ system, one uses a single conserved variable $c$ representing the molar fraction of hydrogen, and one non-conserved variables $\eta_{i}$, called order parameters, for each phase. The value of an order parameter is equal to 1 in the phase that it represents, equal to 0 in other phases or other orientations, and fluctuates between 0 and 1 at the interface. Fig. 1 shows a precipitate in a surrounding matrix, with the corresponding values of the order parameters.

\section{$2.2 \quad$ Total free energy}

The phase field approach is based on minimization of the total free energy, which is given by:

$$
F=\int_{V}\left(f_{\text {chemical }}+f_{\text {interface }}+f_{\text {mechanical }}\right) d V
$$

where $f_{\text {chemical }}$ corresponds to the chemical free energy density. It can be a polynomial function, or a more complicated and physically accurate function. $f_{\text {interface }}$ corresponds to the interfacial free energy, and $f_{\text {mechanical }}$ is the contribution from the mechanical energy, which takes into account the elastic and plastic deformations of the 


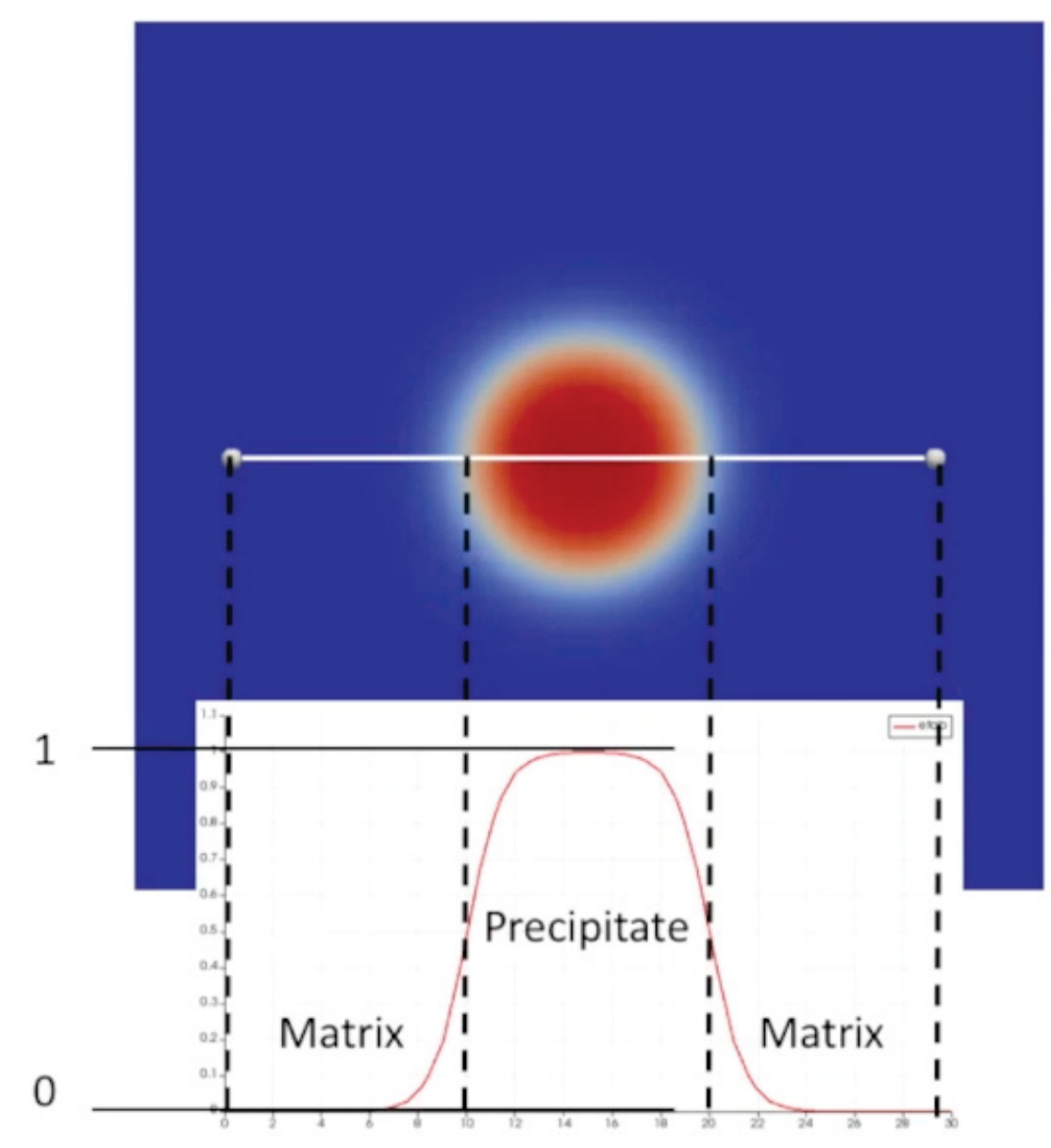

Figure 1 - The order parameters describe the microstructure. An order parameter is linked to the orientation of a phase of the system. It is equal to 1 in a grain of this phase with this orientation, and continuously goes to 0 outside of the grain.

system.

\subsection{Phase field equations}

The evolution of the system is governed by the minimization of the free energy. Nevertheless, conserved and non-conserved parameters evolve in different ways. The spatial and temporal evolution of the microstructure are governed by two coupled equations, the Cahn-Hilliard and the Allen-Cahn equations. 


\subsubsection{Cahn-Hilliard equation, for conserved variables}

The temporal evolution of the conserved variables are determined by the Cahn-Hilliard equation [8]. This equation is derived from the equation of the flux given by the theory of irreversible thermodynamics and the law of mass conservation.

$$
\frac{\partial c}{\partial t}=\nabla \cdot M \nabla\left(\frac{\delta F}{\delta c}\right)
$$

where $M$ is the mobility.

\subsubsection{Allen-Cahn equation, for non-conserved variables}

The temporal evolution of the non-conserved order parameters is described by the AllenCahn equation [9]. This equation is a reaction-diffusion equation, and does not have the constraint of mass conservation like the Cahn-Hilliard equation. The time rate of change of such a parameter is hence directly proportional to its diffusion potential.

$$
\frac{\partial \eta}{\partial t}=-L\left(\frac{\delta F}{\delta \eta}\right)
$$

Where $L$ is a positive kinetic parameter related to the interfacial mobility.

\section{Grand potential model}

\subsection{Generalities}

One of the limitations of the multi-phase field model is the intrinsic coupling between the bulk free energy of the different phases of the system and the interfacial free energy. This coupling often causes the interface to be strictly limited in size, which makes the simulations computationally expensive due to the necessity to define a very fine mesh. The grand potential model does not have any coupling between the bulk free energies and the interfacial free energy, allowing to scale up the interfaces' sizes, thus reducing the computational time of the simulations. The approach is different than the multi- 
phase field model, but Plapp has shown that the two models are perfectly equivalent under certain constraints [10]. The evolution of the system is based on a grand potential functional instead of a free energy functional, and the chemical potential replaces the composition as the fundamental field.

\subsection{Expression of the grand potential}

The grand potential functional is described by the following expression:

$$
\Omega=\int_{V}\left(\omega_{\text {bulk }}+\omega_{\text {grad }}+\omega_{\text {chemical }}+\omega_{\text {elastic }}\right) d V
$$

$\omega_{\text {bulk }}$ represent the bulk grand potential density. $\omega_{\text {grad }}$ corresponds to the contribution of the gradient of the order parameters to the grand potential density. $\omega_{\text {chemical }}$ describes the contribution of the chemical free energy. The last term $\omega_{\text {elastic }}$ takes the elastic contribution to the grand potential into account.

\subsection{Grand potential models equations}

The field variables of the grand potential model are the chemical potential $\mu$ and the order parameters $\eta_{i}$.

Equation 5 governs the evolution of the chemical potential. The first term is analogue to a diffusion term, while the second term corresponds to the effect of a difference in composition at the interfaces. $\chi$ is a generalized susceptibility, $D$ is the solute diffusion coefficient, $\rho$ is the density of solute.

$$
\frac{\partial \mu}{\partial t}=\frac{1}{\chi}\left(\nabla(D(\eta, \mu) \chi(\eta, \mu) \nabla \mu)-\frac{g^{\prime}(\eta)}{2}\left(\rho_{s}(\mu)-\rho_{l}(\mu)\right) \frac{\partial \eta}{\partial t}\right)
$$

The evolution of the order parameters follows equation 6 .

$$
\frac{\partial \eta_{\alpha i}}{\partial t}=-L\left[\frac{\partial \Omega}{\partial \eta_{\alpha i}}\right]
$$

Where $L$ a positive kinetic constant that corresponds to the mobility of the interfaces. 


\section{Coupling with elasticity}

\subsection{Generalities}

The evolution of a system, especially a microstructure, is often dependent on the mechanical properties of the system. The phase field models need to be coupled with an elastic model in order to obtain quantitative results.

\subsection{VTS and KHS models}

There are different possible interpolation schemes that exist to introduce the elastic free energy contribution to the total free energy, or to the grand potential. Two of them are the Voight-Taylor scheme (VTS) [11] and the Khachaturyan's scheme (KHS) [12], and they are not equivalent. The difference lies in the interpolation of the material properties at the interface between two phases. In the VTS model, the elastic energy is interpolated between the two phases. The stress and strain are determined separately in each phase, then the elastic energy is determined for every phase. Only then is the global free elastic energy defined as the interpolation of all the elastic free energy. In the KHS model, the stiffness tensor and the misfit strain are interpolated to create a global stiffness tensor and a global strain. The stress is then determined as a global stress, and the contribution of the elastic free energy is added to the phase field model.

\subsection{Simulations results, and comparison with analytical solu- tion}

The new models coupled with elasticity were verified by comparing the results with analytical solution for a 2 phase system $(\alpha$, and $\beta)$ in different configurations, as was done by Aagesen in [13] for another phase field model. One of these configuration is the bicrystal configuration. Figure 2 shows the geometry of the system and the profile along which the results are plotted. 


\begin{tabular}{|l|l|l|}
\hline$\alpha$ & $\beta$ & $\alpha$ \\
\hline & & \\
\hline
\end{tabular}

Figure 2 - Schematic of the geometry of the domain for the bicrystal configuration. The profile of the results was plotted along the red line.

Fig. 3 shows the profile of the elastic free energy and allows to compare the performances of the different elastic models in the bicrystal configuration. The analytical solution presents, by construction, no excess elastic energy. The VTS and KHS model present respectively a positive excess elastic energy with a large peak at the interface, and a slightly negative excess elastic free energy. The KHS model seems more appropriate then the VTS model for the introduction of the elastic free energy contribution to the grand potential.

\section{Profile of the elastic free energy for different elastic models, and analytical solution}

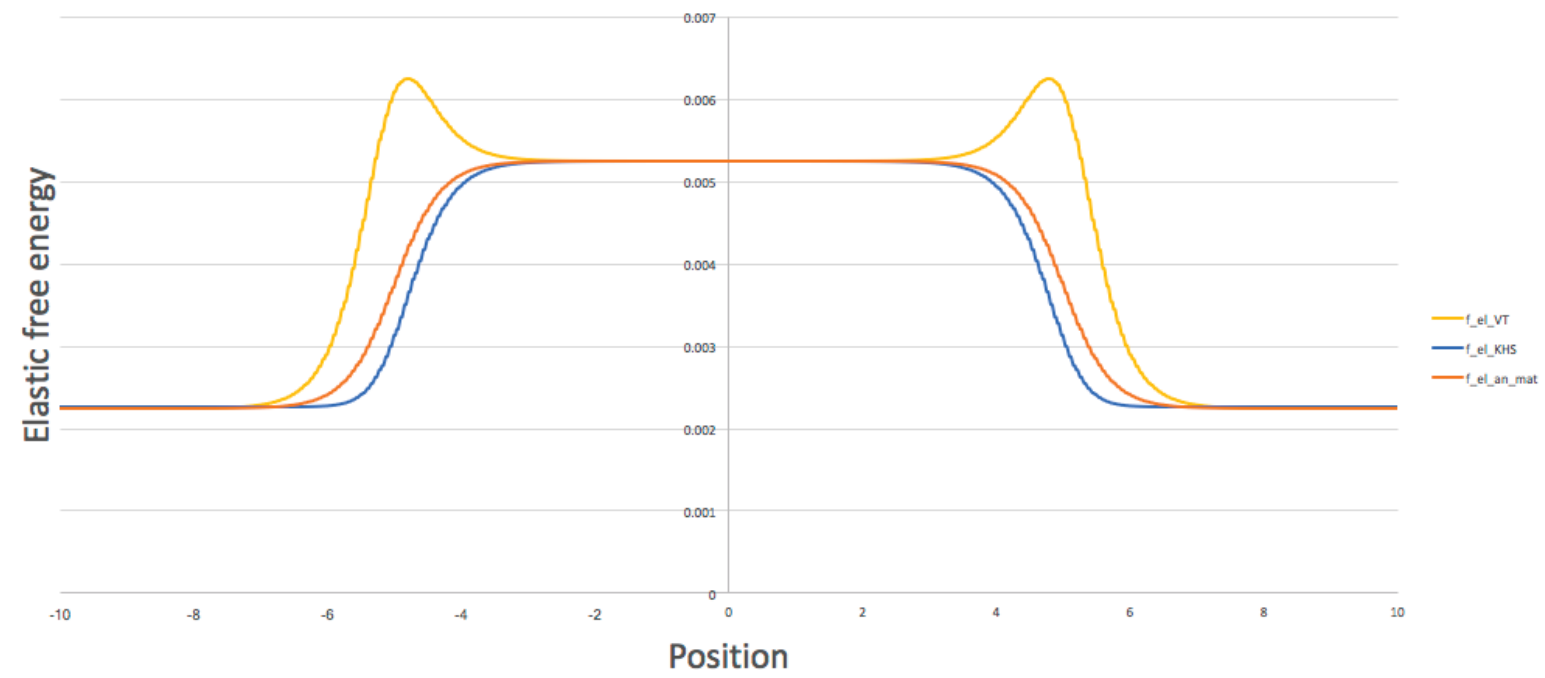

Figure 3 - Comparison of the elastic energy profiles derived using different elastic models (VTS and KHS) with the analytical solution for the bicrystal configuration. The analytical solution, plotted in orange, corresponds to an interpolation of the elastic free energy using the order parameter profile obtained without elasticity. The VTS model overestimates the elastic energy contribution, while the KHS model underestimates it. 


\section{Application to the Zirconium hydride model}

Studying the evolution of the morphology of Zirconium hydride requires to model a 2 phases system. The first phase, called the $\alpha$-Zr phase, corresponds to the $\alpha$ zirconium phase with hydrogen in solid solution. The other phase, called $\delta$ phase represents the hydride phase.

To apply the phase field models coupled with elasticity described in section 3 , the chemical and mechanical properties from the $\alpha$ and $\delta$ phases are introduced in the model, and the parameters of the models are define to reproduce the characteristics of the zirconium hydride model such as the interfacial energy [14].

This model uses expression for the free energies of the hydrides based on results obtained using the density function theory [14]. These free energies shown on Fig. 4 are based on experimental and simulation results on zirconium hydrides. The grand potential densities are easily derived from the free energy densities $\left(\omega_{\nu}(\mu)=f_{\nu}(\mu)-\mu \rho\right)$.

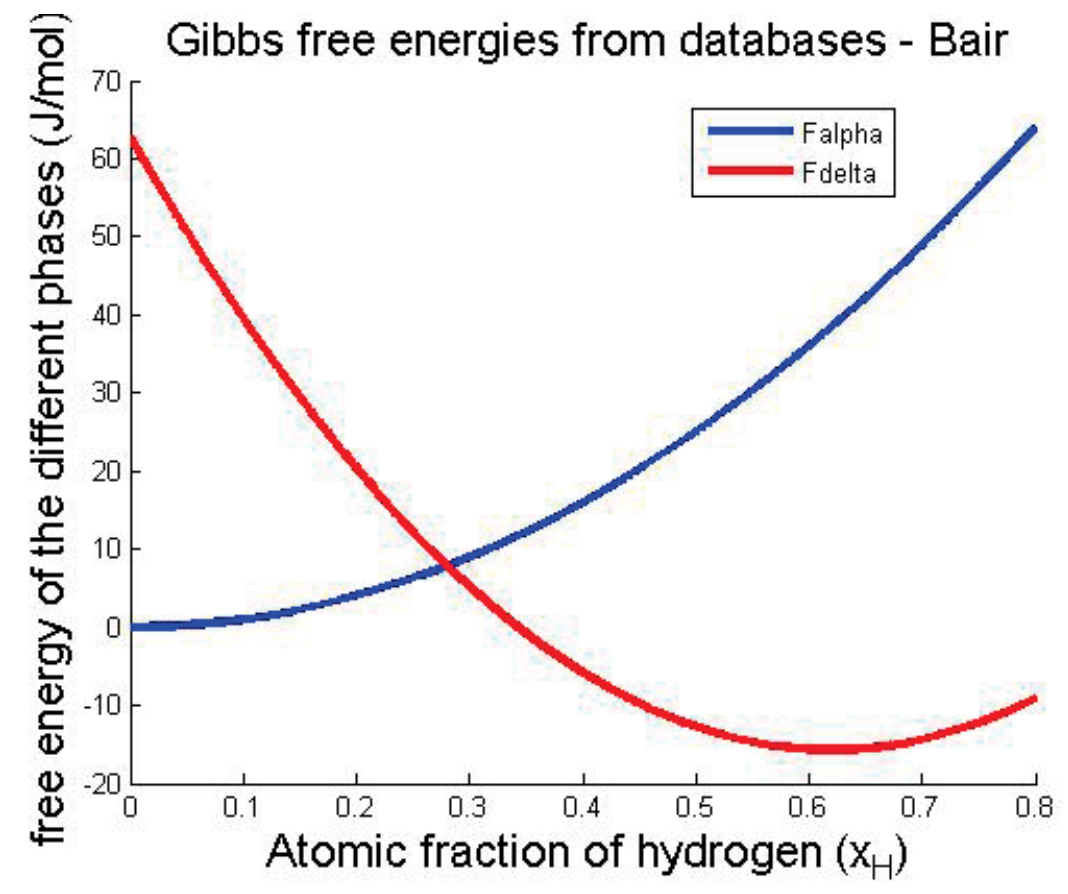

Figure 4 - Gibbs free energies of the different phases of the model.

Mechanical properties of the different phases of the model like the elastic constants 
or the eigenstrain values can be found in the literature [15, [16, [17]. Coupling the zirconium hydride phase field model with the VTS or the KHS model is similar to what has been described in section 1 .

\section{Conclusion and future work}

A multi-phase field model and a grand potential model coupled with elasticity have been developed in MOOSE. These models have been verified by comparing the results to analytical solutions in simple cases, and was then applied to the $\alpha-\mathrm{Zr} / \delta-\mathrm{ZrH}_{1.66}$ system. Different elastic models were implemented, and this study has confirmed the fact that the KHS elastic model provides more accurate results than the VTS model.

The inherent decoupling of the bulk free energy from the interfacial energy that the grand potential model presents makes it very interesting compared with other phase field models. The interface thicknesses can be defined larger than in other models, allowing the use of coarser mesh in simulations, and could thus provide faster results. This could allow to observe clear orientation of the hydride along the basal plane, which was difficult before due to the computational costs of simulations when using the multi-phase field model.

Future work should focus on adding plasticity to the phase field models coupled with elasticity to obtain quantitative results for the $\alpha-\mathrm{Zr} / \delta-\mathrm{ZrH}_{1.66}$ system that could be compared with experimental results. 


\section{References}

[1] Kimberly B. Colas. Fundamental experiments on hydride reorientation in zircaloy. Ph.D. Thesis, page 309, 2012.

[2] Kimberly B. Colas, Arthur T. Motta, Mark R. Daymond, and Jonathan D. Almer. Effect of thermo-mechanical cycling on zirconium hydride reorientation studied in situ with synchrotron X-ray diffraction. Journal of Nuclear Materials, 440(1-3):586-595, September 2013.

[3] Mahmut N. Cinbiz, Donald A. Koss, Arthur T. Motta, Jun-Sang Park, and Jonathan D. Almer. In situ synchrotron X-ray diffraction study of hydrides in Zircaloy-4 during thermomechanical cycling. Journal of Nuclear Materials, 487:247-259, April 2017.

[4] X.Q. Ma, S.Q. Shi, C.H. Woo, and L.Q. Chen. The phase field model for hydrogen diffusion and gamma-hydride precipitation in zirconium under non-uniformly applied stress. Mechanics of Materials, 38, 2006.

[5] Nele Moelans, Bart Blanpain, and Patrick Wollants. An introduction to phase-field modeling of microstructure evolution. Calphad, 32(2):268-294, June 2008. very clear, gives a detailed physical and mathematical point of view.

[6] Long-Qing Chen. Phase-Field Models for Microstructure Evolution. Annual Review of Materials Research, 32(1):113-140, 2002.

[7] Ingo Steinbach. Phase-field models in materials science. Modelling and Simulation in Materials Science and Engineering, 17(7):073001, 2009.

[8] John W. Cahn and John E. Hilliard. Free Energy of a Nonuniform System. I. Interfacial Free Energy. The Journal of Chemical Physics, 28(2):258-267, February 1958. 
[9] J. Cahn and S. Allen. A Microscopic Theory for Domain Wall Motion and its Experimental Verification in Fe-Al Alloy Domain Growth Kinetics. Journal de Physique Colloques, 38(C7):C7-51-C7-54, 1977.

[10] Mathis Plapp. Unified derivation of phase-field models for alloy solidification from a grand-potential functional. Physical Review E, 84(3):031601, September 2011.

[11] Kais Ammar, Benoit Appolaire, Georges Cailletaud, and Samuel Forest. Combining phase field approach and homogenization methods for modelling phase transformation in elastoplastic media. Revue européenne de mecanique numerique, 18:485-523, 2009.

[12] Armen G Khachaturyan. Theory of Structural Transformations in Solids. Dover Publications, Newburyport, 2013. OCLC: 900346426.

[13] Larry K. Aagesen, Daniel Schwen, Karim Ahmed, and Michael R. Tonks. Quantifying elastic energy effects on interfacial energy in the Kim-Kim-Suzuki phasefield model with different interpolation schemes. Computational Materials Science, 140:10-21, December 2017.

[14] Jacob Bair, Mohsen Asle Zaeem, and Daniel Schwen. Formation path of delta hydrides in zirconium by multiphase field modeling. Acta Materialia, 123:235-244, January 2017.

[15] Zhihua Xiao, Mingjun Hao, Xianghua Guo, Guoyi Tang, and San-Qiang Shi. A quantitative phase field model for hydride precipitation in zirconium alloys: Part II. Modeling of temperature dependent hydride precipitation. Journal of Nuclear Materials, 459:330-338, April 2015.

[16] E. S. Fisher and C. J. Renken. Single-Crystal Elastic Moduli and the hcp \ensuremath $\{\backslash$ rightarrow $\}$ bcc Transformation in Ti, Zr, and Hf. Physical Review, 135(2A):A482-A494, July 1964. 
[17] P. A. T. Olsson, A. R. Massih, J. Blomqvist, A. M. Alvarez Holston, and C. Bjerkén. Ab initio thermodynamics of zirconium hydrides and deuterides. Computational Materials Science, 86:211-222, April 2014. 